\title{
SOME REMARKS ON PAULI DATA IN QUANTUM MECHANICS
}

\author{
CHARLES N. FRIEDMAN ${ }^{1}$
}

(Received 23 September 1987)

\begin{abstract}
The problem of determining a square integrable function from its modulus and that of its Fourier transform has been considered in an article by Corbett and Hurst ([1]). In this work we point out an error in one of the main results of the cited article concerning the Pauli uniqueness of real states, provide a proof of uniqueness for non-negative states, and present various related examples and discussion of the problem.
\end{abstract}

\section{Introduction}

Suppose $\psi(x) \in L^{2}(\mathbf{R})$ is a square integrable (complex-valued) function on the real line with Fourier transform

$$
\hat{\psi}(k)=(2 \pi)^{-1 / 2} \int d x e^{i k x} \psi(x) .
$$

In [1], the authors consider the problem of determining $\psi$ up to a constant multiple of modulus one from the data

$$
\{|\psi(x)|,|\hat{\psi}(k)|\} .
$$

We recall some notation and terminology from [1], and introduce some of our own. First, since (1) is unchanged if $\psi$ is replaced by $\alpha \psi$ where $\alpha$ is a constant of modulus one, we can only hope to determine from (1) the ray containing $\psi$ in $L^{2}(\mathbf{R})$. We thus introduce the following terminology: if $f$ and $g$ are square integrable functions, we say " $f$ is a representative of $g$ " if $f=\alpha g$ almost everywhere for some constant $\alpha$ of modulus one. This (equivalence) relation will appear in locutions such as " $\psi$ has a real representative", etc. The data of (1) is termed by

${ }^{1}$ Department of Mathematics, The University of Texas, Austin, Texas, U.S.A.

(C) Copyright Australian Mathematical Society 1989, Serial-fee code 0334-2700/89 
the authors of [1], the Pauli data of $\psi$, and $\psi$ is said to be Pauli unique if only representatives of $\psi$ give rise to the same data almost everywhere. A function satisfying the same Pauli data as $\psi$, and which is not a representative of $\psi$, is called a Pauli partner of $\psi$.

The question of when Pauli uniqueness occurs was raised by Pauli (see [1] for references) in the context of quantum mechanics. If, for example, $\psi(x)$ is the wave function for a single quantum mechanical particle in one dimension, one could ask whether the state $\psi$ (i.e. the corresponding ray $\{\alpha \psi|| \alpha \mid=1\}$ ) is determined by the position and momentum distributions $|\psi|^{2} d x$ and $|\hat{\psi}|^{2} d k$. We remark that if these are considered as the statistical distributions of position and momentum for an ensemble of classical particles, then the state of this classical system is not completely described due to a lack of information concerning the correlation between positions and momenta. Of course, such an interpretation of these distributions is not correct; nevertheless, in general, Pauli uniqueness does not hold, although non-uniqueness appears to occur with a multiplicity much less extreme than contemplation of the cited classical analogue might indicate.

In [1], the authors claim to prove that if $\psi$ or $\hat{\psi}$ has a real representative and a further regularity condition holds, then $\psi$ is Pauli unique (Theorems 2 and 6 of [1]). In the next section we show this to be incorrect. We do prove, however, that if $\psi$ or $\hat{\psi}$ has a non-negative representative (no further regularity conditions necessary) then $\psi$ is Pauli unique. We also construct some examples of non-unique states and related phenomena and discuss a conjecture concerning the multiplicity of non-uniqueness in general.

\section{Examples of Pauli non-uniqueness and a theorem on uniqueness}

We first construct a class of Pauli non-unique states. Suppose $f \in L^{2}(\mathbf{R})$. Then an elementary calculation shows that with $g(x)=\bar{f}(-x+2 b), b \in \mathbf{R}$, we have

$$
\hat{g}(k)=e^{-2 i b k} \overline{\hat{f}(k)}
$$

which yields the following.

PROPOSITION 1. If $f \in L^{2}(\mathbf{R})$ and $|f|$ is symmetric about $x=b$ (i.e. $|f(-x+2 b)|=|f(x)|)$, then provided that $g(x)=\vec{f}(-x+2 b)$ is not a representative of $f, f$ and $g$ are Pauli partners.

An analogous result holds if $\mathbf{R}$ is replaced by $\mathbf{R}^{n}$. In this case one may even replace the transform $x \rightarrow-x+2 b$ by an element of the inhomogeneous orthogonal group. One may of course construct examples of Pauli partners by starting with an $f$ for which $|\hat{f}|$ is symmetric about some $b$. We conjecture that 
the construction described in the proposition is canonical in the sense that all examples of Pauli partners arise in some simple way from f's for which $|f|$ or $|\hat{f}|$ is symmetric about some point. However, the precise formulation of such a conjecture seems elusive. For example, it is not clear to this author whether or not there exists a nonzero $f \in L^{2}(\mathbf{R})$ with $|f|$ symmetric about $b,|\hat{f}|$ symmetric about $b^{\prime}$ and $b, b^{\prime}$ both non-zero. (Note that if $f$ itself is symmetric about $b$, then $|\hat{f}|$ is symmetric about 0 and hence $|\hat{f}|$ is not symmetric about $b^{\prime} \neq 0$ since $|\hat{f}| \in L^{2}$.)

The construction of the proposition easily yields examples of Pauli partners $f, g$ which are real and with arbitrary smoothness.

COROLLARY 1. Suppose $f$ and $g$ are nonzero real-valued square-integrable functions on $\mathbf{R}$ with disjoint support and such that $f$ is even and $g$ is odd. Let $\psi=f+g$. Then $\psi(x)$ and $\psi(-x)$ are Pauli partners, hence, $\psi$ is not Pauli unique.

ProOF. $|\psi|$ is even, but $\psi$ has no definite parity.

Since $\psi$ could be taken to be $C^{\infty}$ with compact support for example, this shows that Theorem 2 of [1] is incorrect. (The error in the proof occurs when it is concluded that $\int \varphi\left(g^{\prime}\right)^{2} \varphi d x=0$ implies $g$ is constant almost everywhere; in fact the vanishing of the integral only implies that $g$ is constant almost everywhere on each component of the set where $\varphi \neq 0$.)

We now turn to the question of Pauli uniqueness for functions $\psi \in L^{2}$ with a non-negative representative. Before proving a result, it is convenient to rewrite the data (1) in terms of $\psi$ only. Since the Fourier transform of a product is proportional to the convolution of the Fourier transforms and the transform of $\bar{f}(x)$ is $\overline{\hat{f}(-k)}$, taking the inverse Fourier transform of $|\hat{\psi}|^{2}$, it follows that the data of (1) is equivalent to

$$
\left\{|\psi(x)|, \int \psi(x+h) \bar{\psi}(x) d x\right\}, \quad h \in \mathbf{R} .
$$

If we put $\psi(x)=|\psi(x)| e^{i \theta(x)}$, then the second entry in (3) is

$$
\int|\psi(x+h) \psi(x)| e^{\imath \Delta \theta} d x ; \quad \Delta \theta=\theta(x+h)-\theta(x) .
$$

THEOREM 1. Suppose $f \in L^{2}(\mathbf{R})$ and either $f$ or $\hat{f}$ has a non-negative representative. Then $f$ is Pauli unique.

PROOF. We consider only the case that $f$ has a non-negative representative, the alternative case being completely analogous, and we may assume without loss of generality that $f \geq 0$. 
If $g$ has the same Pauli data as $f$, then writing $g(x)=f(x) e^{x \theta(x)}$ (assume $\theta(x)=0$ when $f(x)=0$ ), we have

$$
\int f(x+h) f(x)\left(e^{i \Delta \theta}-1\right) d x=0, \quad \text { for all } h .
$$

Since $f \geq 0$, the integrand has real part $\leq 0$, hence equals 0 almost everywhere, and it then follows that the imaginary part of the integrand equals 0 almost everywhere also $(\sin \theta=0$ when $\cos \theta=1)$. Therefore we have that

$$
f(x+h) f(x)\left|e^{i \Delta \theta}-1\right|=0 \quad \text { almost everywhere, for all } h \in \mathbf{R} .
$$

An application of Fubini's Theorem ([3]) then shows that for almost all $x$,

$$
f(x+h) f(x)\left|e^{\imath \Delta \theta}-1\right|=0 \quad \text { for almost all } h .
$$

From (7) it follows that $e^{2 \theta(x)}$ is constant almost everywhere on the set where $f \neq 0$, and $g$ is a representative of $f$.

\section{Complements}

In this section, we make some further remarks concerning some of the results discussed previously, mention some related or analogous problems, etc.

First we mention that a slight change in the construction used in Corollary 1 of the previous section yields a simple construction of distinct probability measures with identical moments. Suppose that we have functions $\psi_{1}, \ldots, \psi_{n}$ which are each $C^{\infty}$, have mutually disjoint compact supports, and are such that for each $i$, either $\psi_{i} \geq 0$ or $\psi_{i} \leq 0$. For each $i$, suppose that $\varphi_{i}= \pm \psi_{i}$ (sign depending on $i$ ), and let $\psi=\sum_{i} \psi_{i}, \varphi=\sum_{i} \varphi_{i}$. Then evidently we have

$$
\int \psi \psi^{(\ell)} d x=\int \varphi \varphi^{(\ell)} d x
$$

(where $\psi^{(\ell)}$ denotes the $\ell$ th derivative of $\psi$ ) and taking Fourier transforms yields

$$
\int \hat{\psi} k^{\ell} \overline{\hat{\psi}} d k=\int \hat{\varphi} k^{\ell} \overline{\hat{\varphi}} d k
$$

That is, the measures $|\hat{\psi}|^{2} d k$ and $|\hat{\varphi}|^{2} d k$ (which may clearly be normalised to be probability measures) have identical moments. However, these measures are distinct in general (unless, for example, $\varphi$ is a representative of $\psi$ or $\psi(-x+2 b)$ for some $b$, as occurs in the corollary).

We make some comments concerning the multiplicity problem for Pauli data. In the previous section, it was conjectured that the examples of Pauli partners $f, g$ for which $g=\bar{f}(-x+2 b)$ or $\hat{g}=\overline{\hat{f}}(-x+2 b)$ are canonical in some sense. An intriguing observation concerning this conjecture has been made by John Semple 
(a graduate student at The University of Texas, Austin). Namely, suppose $f, g$ are $L^{2}$ functions. Then denoting the $L^{2}$ norm by $\|\cdot\|^{2}$, we have

$$
\begin{aligned}
|(f * g)(y)| & =\left|\int f(-x+y) g(x) d x\right| \\
& \leq\|f\|_{2}\|g\|_{2} .
\end{aligned}
$$

It is well known ([3]) that equality holds in (10) exactly when

$$
\alpha g(x)=\beta \bar{f}(-x+y) \quad \text { for almost all } x,
$$

for constants $\alpha, \beta$ not both 0 . Hence, if we fix an $f \in L^{2}, f \neq 0$, then for $g \in L^{2}$ with $|g|=|f|$,

$$
|(f * g)(y)| \leq\|f\|_{2}^{2}
$$

and equality occurs, i.e. $|(f * g)(\cdot)|$ achieves its maximum possible value at some point $y$, exactly when $g$ is a representative of $\bar{f}(-x+y)$. This is precisely the condition that occurs in the cited conjecture. So far, however, this observation has not led to any further result.

Another possible approach to the multiplicity problem is to analyse the situation for functions in some dense subset of $L^{2}$, e.g. functions which have compact support or are real analytic, step functions, etc. Even these cases seem difficult, however; moreover, it is not at all clear how one might proceed to a general result given some positive result for one of these cases.

There is a finite-dimensional analogue of the problem we have been considering whose solution would be of considerable relevance here. For a vector $a=\left(a_{1}, \ldots, a_{n}\right)$ in the $n$-dimensional complex space $\mathbf{C}^{n}$, we may consider the "Pauli data"

$$
\left\{\left|a_{j}\right|, \sum_{i=1}^{n} a_{\imath+j} \bar{a}_{\imath}\right\} \quad j=1, \ldots, n-1
$$

$\left(a_{k}=0\right.$ if $k<1$ or $k>n$ ), and ask which vectors have the data (13). For $n \leq 3$, this problem is fairly trivial, but for larger $n$ it seems rather complicated and intractable.

We remark here also that the present author's interest in the problem discussed in this article stemmed partly from a curiosity concerning the stochastic formulation of quantum mechanics (for which see [2]). Consider a quantum mechanical particle with wave function at time $t$ given by $\psi_{t}(x)$; the probability distributions of position and momentum are then $\left|\psi_{t}\right|^{2} d x$ and $\left|\hat{\psi}_{t}\right|^{2} d k$. If the position at time $t$ were a stochastic process $X_{t}$, then we would have

$$
P\left(X_{t} \in A\right)=\int_{A}\left|\psi_{t}\right|^{2} d x
$$

Now the probability that an observation to determine whether the particle is in the set $A$ at time $t$ yields an affirmative answer is given by the right side of 
(14), but in the usual formulation of quantum mechanics, there are of course no $X_{t}$ 's. For example, in the usual formulation, joint and conditional probabilities such as $P\left(X_{t_{1}} \in A_{1}, X_{t_{2}} \in A_{2}\right)$ and $P\left(X_{t_{2}} \in A_{2} \mid X_{t_{1}} \in A_{1}\right), t_{2}>t_{1}$, have no meaning, nor does the equation

$$
P\left(X_{t_{2}} \in A_{2}, X_{t_{1}} \in A_{1}\right)=P\left(X_{t_{2}} \in A_{2} \mid X_{t_{1}} \in A_{1}\right) P\left(X_{t_{1}} \in A_{1}\right) .
$$

It is, however, tempting to contemplate giving a meaning to a conditional probability such as $P\left(X_{t+\Delta t} \in A_{2} \mid X_{t}=x\right)$ as an expression defined in terms of $\left|\psi_{t}\right|^{2} d x$ and $\left|\hat{\psi}_{t}\right|^{2} d k$; the probability of moving from $x$ to some position in $A_{2}$ ought to depend on the momentum distribution at time $t$. A natural question is then whether one can in fact determine (even partially) $\left|\psi_{t+\Delta t}\right|^{2}$ in terms of $\left|\psi_{t}\right|^{2}$ and $\left|\hat{\psi}_{t}\right|^{2}$, thereby possibly making sense of the conditional probability intervening in (15). This would be possible if one could explicitly determine $\psi_{t}$ given $\left|\psi_{t}\right|$ and $\left|\hat{\psi}_{t}\right|$, and one knew the Hamiltonian for the physical process. It is conceivable that even if $\psi_{t}$ and hence $\psi_{t+\Delta t}$ is not uniquely determined by $\left|\psi_{t}\right|$ and $\left|\hat{\psi}_{t}\right|$, nevertheless $\left|\psi_{t+\Delta t}\right|$ is determined by this data, and such a result might be of relevance in understanding the stochastic nature of quantum mechanics.

\section{References}

[1] J. V. Corbett and C. A. Hurst, "Are wave functions uniquely determined by their position and momentum distributions?", J. Austral. Math. Soc. Ser. B 20 (1977) 182-201.

[2] E. Nelson, Quantum fuctuations (Princeton Series in Physics, Princeton University Press, Princeton, N. J., 1985).

[3] W. Rudin, Real and complex analysis (McGraw Hill, New York, 1966). 